\title{
The missing link: Tracing molecular gas in the outer filament of Centaurus A
}

\author{
Raffaella Morganti ${ }^{1,2}$, Tom Oosterloo ${ }^{1,2}$, J. B. Raymond Oonk ${ }^{1,3}$, \\ Francesco Santoro ${ }^{1,2}$, and Clive Tadhunter ${ }^{4}$ \\ 1 ASTRON, the Netherlands Institute for Radio Astronomy, Postbus 2, 7990 AA Dwingeloo, The Netherlands \\ e-mail: morganti@astron.nl \\ 2 Kapteyn Astronomical Institute, University of Groningen, PO Box 800, 9700 AV Groningen, The Netherlands \\ ${ }^{3}$ Leiden Observatory, Leiden University, PO Box 9513, 2300 RA Leiden, The Netherlands \\ ${ }^{4}$ Department of Physics and Astronomy, University of Sheffield, Sheffield, S7 3RH, UK \\ Received 17 May 2016/ Accepted 4 July 2016
}

\section{ABSTRACT}

\begin{abstract}
We report the detection, using observations of the $\mathrm{CO}(2-1)$ line performed with the Atacama Pathfinder EXperiment (APEX), of molecular gas in the region of the outer filament of Centaurus A, a complex region known to show various signatures of an interaction between the radio jet, an $\mathrm{HI}$ cloud, and ionised gas filaments. We detect $\mathrm{CO}(2-1)$ at all observed locations, which were selected to represent regions with very different physical conditions. The $\mathrm{H}_{2}$ masses of the detections range between $0.2 \times 10^{6}$ and $1.1 \times 10^{6} M_{\odot}$, for conservative choices of the $\mathrm{CO}$ to $\mathrm{H}_{2}$ conversion factor. Surprisingly, the stronger detections are not coincident with the $\mathrm{H}$ I cloud, but instead are in the region of the ionised filaments. We also find variations in the widths of the $\mathrm{CO}(2-1)$ lines throughout the region, with broader lines in the region of the ionised gas, i.e. where the jet-cloud interaction is strongest, and with narrow profiles in the H I cloud. This may indicate that the molecular gas in the region of the ionised gas has the momentum of the jet-cloud interaction encoded in it, in the same way as the ionised gas does. These molecular clouds may therefore be the result of very efficient cooling of the down-stream gas photo- or shock-ionised by the interaction. On the other hand, the molecular clouds with narrower profiles, which are closer to or inside the $\mathrm{H}$ I cloud, could be pre-existing cold $\mathrm{H}_{2}$ cores which manage to survive the effects of the passing jet.

Key words. galaxies: active - galaxies: individual: Centaurus A - ISM: jets and outflows - radio lines: galaxies
\end{abstract}

\section{Introduction}

Radio-loud active galactic nuclei (AGN) are known to inject energy into the surrounding interstellar medium (ISM) via plasma jets. The impact this has on the host galaxy is relevant on both large and small scales (see e.g. McNamara \& Nulsen 2012; Morganti et al. 2013). The induced compression - and subsequent cooling - of gas disturbed by the transit of a radio jet can induce the formation of new stars (e.g. van Breugel \& Dey 1993; Dey et al. 1997; Oosterloo \& Morganti 2005; Croft et al. 2006), but AGN-driven gas outflows can also occur which remove, or at least redistribute, the cold gas and/or create highly turbulent conditions which inhibit star formation (e.g. Alatalo et al. 2011; Combes et al. 2013; García-Burillo et al. 2014; Morganti et al. 2013, 2015; Guillard et al. 2015).

One of the important results of recent work is that molecular gas often is the most massive component in jet-cloud interactions (e.g. Feruglio et al. 2010; Alatalo et al. 2011; Cicone et al. 2014; Morganti et al. 2015). Thus, tracing this phase of the gas provides key information on how these processes work and how they depend on the properties of the gas and of the radio jet.

When it comes to studying the interaction between a radio jet and the ISM of the host galaxy, the nearest radio-loud AGN, Centaurus A (Cen A, $D=3.8 \mathrm{Mpc}$, Harris et al. 2010), should be considered a prime target. In this galaxy, about $15 \mathrm{kpc} N E$ from its centre, the so-called outer filament region presents an extremely intriguing situation. Past studies of this region have revealed many signatures of an ongoing interaction between the radio jet and gas clouds (see Morganti 2010 for a review) and over the years a wealth of data has been collected on this region, covering almost all wavebands. However, although it is such a well-studied region, very little information is available about the molecular gas in and around the outer filament, and this has motivated the $\mathrm{CO}(2-1)$ observations with the Atacama Pathfinder EXperiment (APEX) presented in this paper.

\section{Special region: the outer filament}

The outer filament was discovered as a filament of highly ionised gas outside the optical body of Cen A, well aligned with the radio jet of Cen A (Blanco et al. 1975; Graham \& Price 1981; Morganti et al. 1991, 1999; Morganti 2010). Later work identified regions of ongoing star formation in the filament (Mould et al. 2000; Rejkuba et al. 2001), while bright UV emission was detected with GALEX (Neff et al. 2015). About $2 \mathrm{kpc}$ west of the ionised gas, a large H I cloud was found (Schiminovich et al. 1994), likely the remnant of a major accretion by Cen A. In this H I cloud, at locations closest to the radio jet, H I with velocities very different from those of the regular rotation of the $\mathrm{HI}$ cloud around Cen A was detected (Oosterloo \& Morganti 2005), which was taken as evidence that the radio jet is affecting the $\mathrm{HI}$ cloud at those locations. This is further confirmed by integral field spectroscopy with VIMOS and MUSE (Santoro et al. 2015a,b), showing the presence of even more disturbed kinematics in the ionised gas. The MUSE data reveal three morphologically and kinematically distinct components in the ionised gas, which are thought to correspond to different stages in the jet-cloud interaction. The data also show that, overall, the ionisation of the gas is due to ionising photons from the AGN, but that locally star formation also plays a role (Santoro et al. 2016). Interestingly, the rate at which the available gas reservoir is turned into stars is low, possibly connected to increased turbulence powered by injection 
Table 1. Locations of the APEX pointing positions and the noise of the spectra.

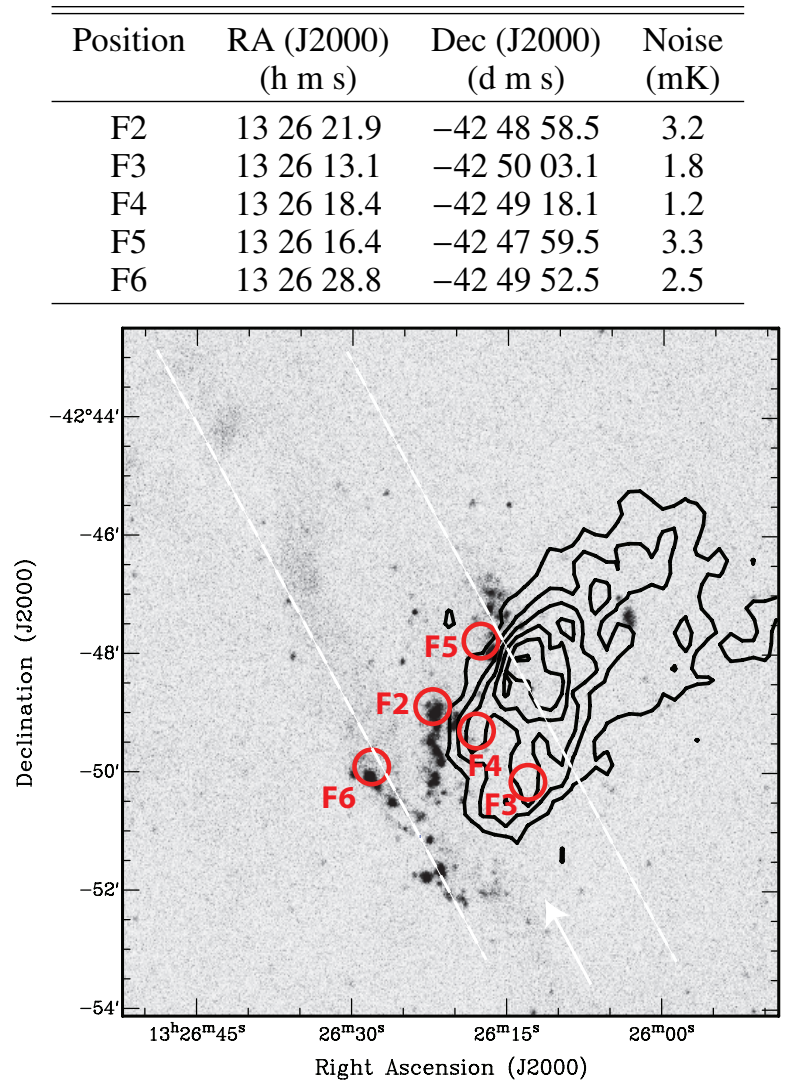

Fig. 1. Locations of the APEX pointings (see Table 1) on the GALEX FUV image of the outer filament (Neff et al. 2015). The black contours give the distribution of the $\mathrm{H}$ I cloud with contour levels $1,4,7,10,13$, and $16 \times 10^{20} \mathrm{~cm}^{-2}$ (Oosterloo \& Morganti 2005). The dashed lines approximately delineate the path of the radio jet (Morganti et al. 1999); the arrow shows the flow direction of the jet.

of kinetic energy by the jet (Salomé et al. 2016a). The Balmer decrement $(\mathrm{H} \alpha / \mathrm{H} \beta)$ across the MUSE fields confirms that the ionised gas is dusty. The presence of very cold dust $(T \sim 13 \mathrm{~K})$ in and around the outer filament is also seen in Herschel data (Auld et al. 2012).

The above shows that the region around the outer filament is very rich in phenomena and that it shows the full complexity of the interaction of a jet with a large gas cloud. This interaction is clearly stirring up a gas cloud and is destroying it (partially), but at the same time star formation is happening with low efficiency.

To complete this picture, information on a key component is missing: the molecular gas. The only observations available around this region are those done with SEST by Charmandaris et al. (2000) of locations in the H I cloud and ALMA observations of a single location just north of the H I cloud (Salomé et al. 2016a). They have detected molecular gas associated with the northern region of this cloud and estimate that the molecular gas is the most massive component at the observed locations. These observations, however, only sample regions relatively distant $(\sim 3 \mathrm{kpc})$ from the outer filament and from the region with anomalous H I velocities. Here we report observations which have a more complete coverage of the outer filament.

\section{APEX observations}

Observations with the Atacama Pathfinder EXperiment (APEX) $12 \mathrm{~m}$ antenna were conducted between 7 and 10 April 2016, using the APEX-1 instrument with the XFFTS backend tuned to $230.5486 \mathrm{GHz}$, the frequency of $\mathrm{CO}(2-1)$ corresponding to the velocity of the gas in the outer filament $\left(V_{\text {hel }} \sim 400 \mathrm{~km} \mathrm{~s}^{-1}\right)$. Five pointing positions were selected to cover different regions across the outer filament in order to sample very different conditions of the gas and related phenomena. Their locations are shown in Fig. 1 and are listed in Table 1. Positions F3 and F4 were chosen to lie in the regularly rotating part of the H I cloud in order to sample the quiescent H I gas. To sample a location in the main ionised filament where the gas is clearly affected by the jet-cloud interaction, we have chosen a position (F6) where the ionised gas consists of at least two distinct kinematically and morphologically distinct components (Santoro et al. 2015b). Finally, locations F2 and F5 lie just outside the H I cloud at locations where some recent star formation has occurred.

The observations were done in good weather conditions (for these frequencies). The precipitable water vapour (PWV) was between 2.4 and $3.3 \mathrm{~mm}$. The observations were made using 32768 channels covering a total band of $2.5 \mathrm{GHz}$ $\left(\sim 2500 \mathrm{~km} \mathrm{~s}^{-1}\right)$ with a velocity resolution of $0.076 \mathrm{~km} \mathrm{~s}^{-1}$. However, the final spectra were smoothed to $10 \mathrm{~km} \mathrm{~s}^{-1}$ bins. The data were reduced with the CLASS software from the Gildas package $^{1}$ using the standard scripts provided by ESO. From the individual scans of each of the XFFTS units a linear baseline was subtracted before adding all spectra. The noise levels of the spectra are listed in Table 1 . Table 2 presents the parameters of the $\mathrm{CO}(2-1)$ profiles for the five positions derived from Gaussian fits. At the frequency of our observations, the spatial resolution of APEX is $\sim 30^{\prime \prime}(\sim 0.5 \mathrm{kpc})$ and the gain is $39 \mathrm{Jy} \mathrm{K}^{-1}$.

\section{4. $\mathrm{CO}(2-1)$ in the region of the outer filament}

The first important result (Fig. 2 and Table 2) is that we detect $\mathrm{CO}(2-1)$ at all five locations spread over the entire region: in the $\mathrm{H}$ I cloud (F3 and F4), in the main region of ionised gas (F6), but also at locations in between the $\mathrm{H}$ I cloud and the ionised gas (F2 and F5). The first direct consequence is that the molecular gas is the only gas tracer that is present inside the H I cloud, in the ionised filament, and in between the two, while H I and ionised gas are detected in mutually excluding locations. This underlines, as in other cases of jet-cloud interactions, that the molecular gas is a crucial component in such phenomena.

Although limited by the small number of pointings, a trend can be seen in the width of the $\mathrm{CO}(2-1)$ profile. The widths increase going from west to east, from inside the H I cloud to outside it, and finally to the region of ionised gas. This mirrors what is seen in the HI (which has narrow profiles only $\sim 20 \mathrm{~km} \mathrm{~s}^{-1}$ wide; Oosterloo \& Morganti 2005) and the ionised gas (with much more complex kinematics with line widths $>100 \mathrm{~km} \mathrm{~s}^{-1}$; Morganti et al. 1991; Santoro et al. 2015b). The velocities and widths of the profiles F3, F4, and F5 are consistent with those of the regularly rotating part of the $\mathrm{H}$ I cloud and with what was obtained by Charmandaris et al. (2000) for a region near F5. In contrast, the detections towards and in the region of the ionised filament (F2 and F6) are much broader and show gas at velocities corresponding to the high-velocity, disturbed components of the ionised gas (see Santoro et al. 2015a) and are deviating from the extrapolation of the regularly rotating $\mathrm{HI}$. The profile at F6 is broadest with a $F W H M=72 \mathrm{~km} \mathrm{~s}^{-1}$, is asymmetric and shows some indications of having multiple components. The possible presence of multiple components at position F6 may be related to the presence of different kinematical components seen in the

1 http://wWw . iram. fr/IRAMFR/GILDAS 
R. Morganti et al: The missing link: Tracing molecular gas in the outer filament of Centaurus A

Table 2. Parameters of the $\mathrm{CO}(2-1)$ profiles derived using Gaussian fits, derived molecular masses, and ratios of molecular to atomic mass for the five positions.

\begin{tabular}{crrrrrr}
\hline \hline Position & \multicolumn{1}{c}{$\begin{array}{c}\text { Peak } \\
(\mathrm{mK})\end{array}$} & $\begin{array}{c}\text { Velocity } \\
\left(\mathrm{km} \mathrm{s}^{-1}\right)\end{array}$ & \multicolumn{1}{c}{$\begin{array}{c}\text { Dispersion } \\
\left(\mathrm{km} \mathrm{s}^{-1}\right)\end{array}$} & $\begin{array}{c}\text { Integrated flux } \\
\left(\mathrm{mK} \mathrm{km} \mathrm{s}^{-1}\right)\end{array}$ & \multicolumn{1}{c}{$\begin{array}{c}\mathrm{H}_{2} \text { mass } \\
\left(M_{\odot}\right)\end{array}$} & $\mathrm{H}_{2} / \mathrm{H} \mathrm{I}$ \\
\hline F2 & $22.3 \pm 1.7$ & $346.7 \pm 1.5$ & $18.0 \pm 1.5$ & $1007.7 \pm 114.1$ & $9.5 \times 10^{5}$ & $>4.8$ \\
F3 & $17.0 \pm 1.0$ & $332.1 \pm 0.6$ & $8.8 \pm 0.6$ & $374.2 \pm 31.9$ & $3.5 \times 10^{5}$ & 0.4 \\
F4 & $8.7 \pm 0.8$ & $322.7 \pm 1.0$ & $9.7 \pm 1.0$ & $210.7 \pm 28.8$ & $2.0 \times 10^{5}$ & 0.2 \\
F5 & $39.8 \pm 3.0$ & $340.1 \pm 0.8$ & $9.3 \pm 0.8$ & $932.3 \pm 108.8$ & $8.8 \times 10^{5}$ & $>4.4$ \\
F6 & $15.0 \pm 1.1$ & $335.4 \pm 2.6$ & $30.8 \pm 2.6$ & $1154.7 \pm 131.0$ & $10.9 \times 10^{5}$ & $>5.4$ \\
F6-1 & $19.0 \pm 1.2$ & $316.8 \pm 1.1$ & $11.0 \pm 1.0$ & $523.6 \pm 58.1$ & $4.9 \times 10^{5}$ & \\
F6-2 & $12.3 \pm 0.9$ & $359.6 \pm 2.3$ & $18.7 \pm 2.4$ & $577.6 \pm 84.0$ & $5.5 \times 10^{5}$ & \\
\hline
\end{tabular}

Notes. For position F6 the results of a two-Gaussian fit are also given and are labelled F6-1 and F6-2. The molecular masses have been estimated using $\mathrm{CO}(2-1) / \mathrm{CO}(1-0)=0.55$ (Charmandaris et al. 2000) and a conversion factor of $\alpha_{\mathrm{CO}}=1.5 M_{\odot}\left(\mathrm{K} \mathrm{km} \mathrm{s}^{-1} \mathrm{pc}^{2}\right)^{-1}$. H I masses are derived from the data presented in Oosterloo \& Morganti (2005) using the same aperture as the APEX beam.

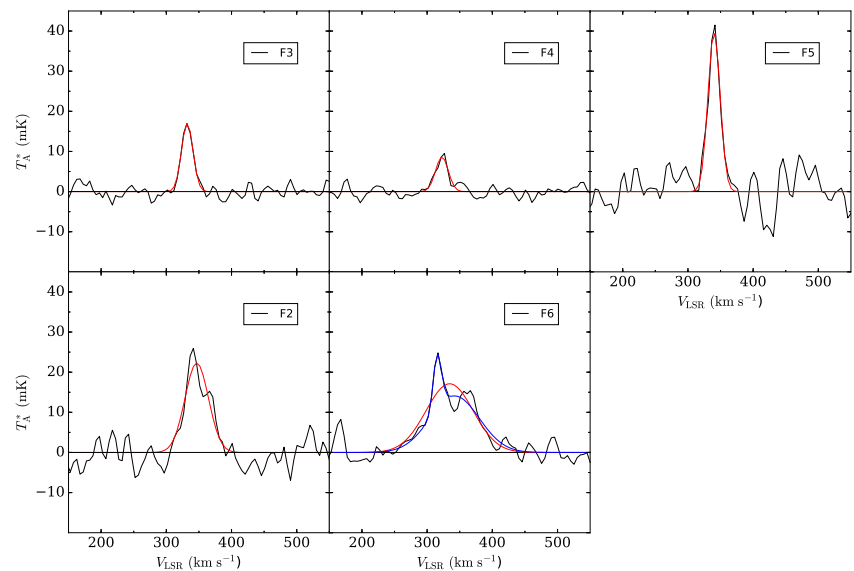

Fig. 2. $\mathrm{CO}(2-1)$ spectra for the observed locations. Red lines show the single-component Gaussian fits, while the blue line for F6 shows the double-Gaussian fit.

ionised gas, in which case it would imply a close connection between the two phases.

It has been noted by several groups that the star formation rate in the outer filament is low and that the rate at which the available cold gas is turned into stars is also low (Mould et al. 2000; Rejkuba et al. 2004; Oosterloo \& Morganti 2005; Salomé et al. 2016a). Salomé et al. (2016a) suggest that turbulence due to kinetic energy injection from the AGN jet leads to molecular gas reservoirs not forming stars efficiently and to quenching of star formation. The differences in line widths we detect do indeed suggest that turbulence is increased due to the jet-cloud interaction. However, star formation only occurs in the region outside the $\mathrm{HI}$ cloud, albeit at low rates, while it seems to be completely absent in the region inside the H I cloud. It therefore seems that the star formation that is occurring is not being quenched, but instead is stimulated by the passage of the radio jet. It is, on the other hand, rather puzzling that no star formation is occurring in the H I cloud where there are no indications that conditions are particularly adverse to star formation. The relative locations of the ionised gas and the $\mathrm{H}$ I are also suggestive that the jet plays a role in the star formation (see Fig. 1). The general possibility of jet-induced star formation has been considered by theoretical models (Mellema et al. 2002; Fragile et al. 2004; Gaibler et al. 2012), but no study has been made for conditions similar to those of the outer filament.

In order to derive molecular masses from our $\mathrm{CO}(2-1)$ detections, we have made assumptions about the $\mathrm{CO}(2-1) / \mathrm{CO}(1-0)$ ratio and the conversion factor CO-to$\mathrm{H}_{2}$. For the former we have assumed the factor obtained by
Charmandaris et al. (2000) for their Shell S1 region, which is located close to $\mathrm{F} 5$ : $\mathrm{CO}(2-1) / \mathrm{CO}(1-0)=0.55$. This may not be correct for all regions covered by our observations and the differences in total line flux between the positions inside and outside the H I cloud could partly be due to different ratios at different locations with different conditions. However, until observations of more transitions are available, it is difficult to apply a varying ratio.

The choice of the conversion factor for $\mathrm{CO}$ to $\mathrm{H}_{2}$ requires some considerations. Charmandaris et al. (2000) have used a standard value, i.e. $\alpha_{\mathrm{CO}}=4.6 M_{\odot}\left(\mathrm{K} \mathrm{km} \mathrm{s}^{-1} \mathrm{pc}^{2}\right)^{-1}$. However, more conservative assumptions have been used for detections in radio AGN. For example, Evans et al. (2005) and Smolčić \& Riechers (2011) have used $\alpha_{\mathrm{CO}}=1.5 M_{\odot}$ $\left(\mathrm{K} \mathrm{km} \mathrm{s}^{-1} \mathrm{pc}^{2}\right)^{-1}$ for $\mathrm{CO}$ detected in radio galaxies. In Table 2 we list the estimated molecular gas masses using the more conservative assumption. Nevertheless, the relatively low metallicity of the gas may suggest that larger conversion factors would be more realistic, resulting in higher values for the $\mathrm{H}_{2}$ masses. However, an even more complex scenario is conceivable where differences in conversion factors exist between different regions owing to their very different physical conditions (e.g. velocity structure of the gas, influence of the jet). Ignoring such complications (which can only be addressed by more detailed observations) and using the more conservative assumption, the masses of the molecular gas range between $0.2 \times 10^{6}$ and $1.1 \times 10^{6} M_{\odot}$ and, like the kinematics, they show differences between the regions coincident with the $\mathrm{HI}$, having the lower $\mathrm{H}_{2}$ masses, and the ones outside, showing higher $\mathrm{H}_{2}$ masses. Again we want to note that the apparent difference in masses between inside and outside the H I cloud could be due in part to not taking different physical conditions into account. A direct comparison of the masses derived above with those of the detections of Charmandaris et al. (2000) is not possible because of the larger area covered by their mosaic observation. However, the peak fluxes and widths of the profiles shown by Charmandaris et al. (2000) are very similar to those of our spectra.

In Table 2 we also present the ratio between molecular and atomic gas masses, $M_{\mathrm{H}_{2}} / M_{\mathrm{HI}}$, where we have derived the $\mathrm{HI}$ masses from the data of Oosterloo \& Morganti (2005) using an aperture of the same size as the APEX beam for each position. We find that for the two regions coincident with the H I cloud this ratio is $\sim 0.3$ while we find a much higher lower limit $(>4)$ for the other regions. Charmandaris et al. (2000) derived a value $M_{\mathrm{H}_{2}} / M_{\mathrm{HI}}=0.8$ for their detection in the $\mathrm{HI}$ cloud. Considering the difference in the CO-to-H2 conversion factor used, our results appear to be consistent with theirs. Despite the uncertainties 
in the conversion factors used, the difference in $M_{\mathrm{H}_{2}} / M_{\mathrm{HI}}$ we find is too large to be due to these uncertainties alone. In fact, the distinctive factor for the difference in mass ratios is the lack of $\mathrm{HI}$ at some locations, more so than the differences in molecular mass. We therefore conclude that the large contrast in mass ratios suggests that the atomic hydrogen is much more affected by the jet-cloud interaction than the molecular gas.

\section{Origin and fate of the molecular gas}

One of the main puzzles of our results is the overall presence of molecular gas, despite the large range of conditions characterising this region near Cen A. One scenario to explain the observations is that molecular clouds originally residing inside the H I cloud are not much affected by the jet-cloud interaction, while the more tenuous atomic hydrogen of the H I cloud is. The molecular gas in the H I cloud is likely to be in denser clumps which would, in this scenario, manage to survive the effects of the passing jet. On the other hand, the $\mathrm{H}$ I itself, being less dense, is affected by the jet and is blown away more easily. The H I is ionised while flowing downstream, resulting in high values of $M_{\mathrm{H}_{2}} / M_{\mathrm{HI}}$. The overall kinematics of the molecular clouds would be very similar to that of the H I cloud (or the extrapolation of it to the regions devoid of $\mathrm{HI}$ ). The increased profile widths of the molecular gas down the flow would mean that, although this gas survives, increased turbulence is somehow induced by the interaction. It has been suggested that the increased turbulence causes the low star formation efficiency in the outer filament (Salomé et al. 2016a). This scenario would explain the relative distribution of the different gas phases, the very different ratios $M_{\mathrm{H}_{2}} / M_{\mathrm{HI}}$, and some of the kinematics. This scenario may occur if, as suggested by the overall kinematics of the ionised gas, only a mild interaction occurs between a slowly moving radio jet and the ISM.

An alternative scenario is similar to what is proposed for other cases of jet-cloud interactions (e.g. Tadhunter et al. 2014). Here it is assumed that all the gas (atomic and molecular) gets photo- or shock-ionised when entering the region of the radio jet. However, denser clumps rapidly reform in the wake of the interaction and cool very efficiently, forming new clouds of molecular gas downstream which ultimately start forming stars. In this scenario $\mathrm{HI}$ is only a very short transient phase in the cooling of the gas from ionised to molecular and its column densities are below the detection limit. As a result, the molecular gas is the most massive component in this region because it is the end product of a fast cooling process and it accumulates over time, giving high values for $M_{\mathrm{H}_{2}} / M_{\mathrm{HI}}$. Since the molecular clumps form from the ionised gas, the kinematics of the molecular gas would mirror that of the ionised gas and less that of the atomic gas.

With the available data, it is not easy to distinguish between the two scenarios. The large profile widths measured outside the H I cloud, as well as the possible presence of multiple components in the same velocity range as those seen in the ionised gas, could suggest that the kinematics of the $\mathrm{H}_{2}$ at these locations is similar to that of the ionised gas, which covers a larger velocity range than that of the $\mathrm{H}$ and which consists of separate components. This may indicate that the molecular gas has the momentum of the interaction encoded in it, in a similar way to the ionised gas, which would argue for the second scenario. On the other hand, the narrow width of profile F5 just outside the $\mathrm{H}$ I cloud matches that of F3 and F4 inside it and would better fit the first scenario of exposed, pre-existing cold $\mathrm{H}_{2}$ cores. It might well be that one scenario is more important in one region and the other scenario at other locations, depending on the local strength and geometry of the jet-cloud interaction.
More extensive observations of the molecular gas, including higher resolution imaging, will be required to study this further. Imaging the region at high spatial resolution with ALMA would allow us to investigate the turbulence and velocity structure of the gas on the scales of individual clouds and as a function of position, at very similar resolution to the optical data. This would make it possible to obtain a detailed view of the connection between the different gas phases and of the processes which occur in the jet-cloud interaction in the outer filament of Cen A.

Acknowledgements. We would like to thank Carlos de Breuck and Theresa Nilsson for their support for the observations. This publication is based on data acquired with the Atacama Pathfinder EXperiment (APEX) which is a collaboration between the Max-Planck-Institut für Radioastronomie, ESO, and the Onsala Space Observatory. We acknowledge the use of the GILDAS software for the data reduction. The research leading to these results has received funding from the European Research Council under the European Union's Seventh Framework Programme (FP/2007-2013)/ERC Advanced Grant RADIOLIFE-320745.

Note added in proof. During the evaluation of this manuscript, we became aware of a manuscript by Salomé et al. (2016b) that also discusses $\mathrm{CO}(2-1)$ APEX observations of this region of Centaurus A. It mostly addresses distinct issues but is consistent/compatible with our conclusions.

\section{References}

Alatalo, K., Blitz, L., Young, L. M., et al. 2011, ApJ, 735, 88

Auld, R., Smith, M. W. L., Bendo, G., et al. 2012, MNRAS, 420, 1882

Blanco, V. M., Graham, J. A., Lasker, B. M., \& Osmer, P. S. 1975, ApJ, 198, L63

Charmandaris, V., Combes, F., \& van der Hulst, J. M. 2000, A\&A, 356, L1

Cicone, C., Maiolino, R., Sturm, E., et al., 2014, A\&A, 562, A21

Combes, F., García-Burillo, S., Casasola, V., et al. 2013, A\&A, 558, A124

Croft, S., van Breugel, W., de Vries, W., et al. 2006, ApJ, 647, 1040

Dey, A., van Breugel, W., Vacca, W. D., \& Antonucci, R. 1997, ApJ, 490, 698

Evans, A. S., Mazzarella, J. M., Surace, J. A., et al. 2005, ApJS, 159, 197

Feruglio, C., Maiolino, R., Piconcelli, E., et al. 2010, A\&A, 518, L155

Fragile, P. C., Murray, S. D., Anninos, P., \& van Breugel, W. 2004, ApJ, 604, 74

Gaibler, V., Khochfar, S., Krause, M., \& Silk, J. 2012, MNRAS, 425, 438

García-Burillo, S., Combes, F., Usero, A., et al. 2014, A\&A, 567, A125

Graham, J. A., \& Price, R. M. 1981, ApJ, 247, 813

Guillard, P., Boulanger, F., Lehnert, M. D., et al. 2015, A\&A, 574, A32

Harris, G. L. H., Rejkuba, M., \& Harris, W. E. 2010, PASA, 27, 475

McNamara, B. R., \& Nulsen, P. E. J. 2012, New J. Phys., 14, 055023

Mellema, G., Kurk, J. D., Röttgering, H. J. A. 2002, A\&A, 395, L13

Morganti, R. 2010, PASA, 27, 463

Morganti, R., Robinson, A., Fosbury, R. A. E., et al. 1991, MNRAS, 249, 91

Morganti, R., Killeen, N. E. B., Ekers, R. D., \& Oosterloo, T. A. 1999, MNRAS, 307,750

Morganti, R., Fogasy, J., Paragi, Z., Oosterloo, T., Orienti, M. 2013, Science, 341,1082

Morganti, R., Oosterloo, T., Oonk, J. B. R., Frieswijk, W., \& Tadhunter, C. 2015, A\&A, 580, A1

Mould, J. R., Ridgewell, A., Gallagher, J. S., III, et al. 2000, ApJ, 536, 266

Neff, S. G., Eilek, J. A., \& Owen, F. N. 2015, ApJ, 802, 88

Oosterloo, T. A., \& Morganti, R. 2005, A\&A, 429, 469

Rejkuba, M., Minniti, D., Silva, D. R., \& Bedding, T. R. 2001, A\&A, 379, 781

Rejkuba, M., Greggio, L., \& Zoccali, M. 2004, A\&A, 415, 915

Salomé, Q., Salomé, P., Combes, F., Hamer, S., \& Heywood, I. 2016a, A\&A, 586, A45

Salomé, Q., Salomé, P., Combes, F., \& Hamer, S. 2016b, A\&A, accepted [arXiv: 1605.05986]

Santoro, F., Oonk, J. B. R., Morganti, R., \& Oosterloo, T. 2015a, A\&A, 574, A89 Santoro, F., Oonk, J. B. R., Morganti, R., Oosterloo, T. A., \& Tremblay, G. 2015b, A\&A, 575, L4

Santoro, F., Oonk, J. B. R., Morganti, R., Oosterloo, T. A., \& Tadhunter, C. 2016, A\&A, 570, A37

Schiminovich, D., van Gorkom, J. H., van der Hulst, J. M., \& Kasow, S. 1994, ApJ, 423, L101

Smolčić, V., \& Riechers, D. A. 2011, ApJ, 730, 64

Tadhunter, C., Morganti, R., Rose, M., Oonk, J. B. R., Oosterloo, T. 2014, Nature, 511, 440

van Breugel, W. J. M., \& Dey, A. 1993, ApJ, 414, 563 